ARTICLE

\title{
Reconfigurable electronics by disassembling and reassembling van der Waals heterostructures
}

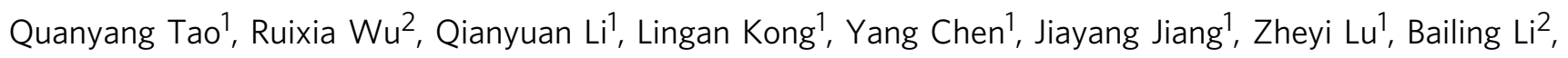

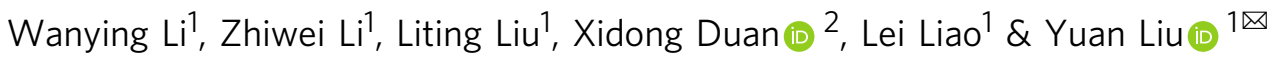

Van der Waals heterostructures ( $v d W H s$ ) have attracted tremendous interest owing to the ability to assemble diverse building blocks without the constraints of lattice matching and processing compatibility. However, once assembled, the fabricated vdWHs can hardly be separated into individual building blocks for further manipulation, mainly due to technical difficulties in the disassembling process. Here, we show a method to disassemble the asfabricated vdWHs into individual building blocks, which can be further reassembled into new vdWHs with different device functionalities. With this technique, we demonstrate reconfigurable transistors from n-type to $\mathrm{p}$-type and back-gate to dual-gate structures through restacking. Furthermore, reconfigurable device behaviors from floating gate memory to Schottky diode and reconfigurable anisotropic Raman behaviors have been obtained through layer re-sequencing and re-twisting, respectively. Our results could lead to a reverse engineering concept of disassembled vdWHs electronics in parallel with state-of-the-art vdWHs electronics, offering a general method for multi-functional pluggable electronics and optoelectronics with limited material building blocks.

\footnotetext{
${ }^{1}$ Key Laboratory for Micro-Nano Optoelectronic Devices of Ministry of Education, School of Physics and Electronics, Hunan University, Changsha, China. 2 State Key Laboratory for Chemo/Biosensing and Chemometrics, College of Chemistry and Chemical Engineering, Hunan University, Changsha, China.

凶email: yuanliuhnu@hnu.edu.cn
} 
W ith dangling-bond-free surface, two-dimensional (2D) layered materials can be isolated and combined to create a wide range of van der Waals heterostructures (vdWHs) without the constraints of conventional lattice matching and processing compatibility ${ }^{1-12}$. With a decade of intensive efforts, vdWHs have become an independent research field, offering a rich playground for both fundamental studies ${ }^{13-17}$ as well as novel device concepts ${ }^{18,19}$. On the other hand, with weak bonding forces towards neighbor layers, the artificial stacked vdWHs could theoretically be disassembled into individual 2D building blocks ${ }^{20}$ and stacked again with different combinations, sequences, angles and hence different device functions, similar as building diverse structures with same pieces of toy bricks ${ }^{3}$. However, this possibility has not been demonstrated and could be largely attributed to technical difficulties in the disassembling process, greatly limiting vdWHs for high-performance devices as well as for investigating fundamental physics. For example, twisting two graphene monolayers into magic angle $\left(1.1^{\circ}\right)$ have enabled unique correlated insulator behavior and superconductivity ${ }^{21,22}$, and similarly, twisting two monolayers of transition metal dichalcogenides have demonstrated tunable moiré potentials inside hetero-bilayers with designable optical and electronic states ${ }^{23-26}$. However, the rotation angle of conventional vdWHs can not be changed once assembled. To identify the accurate angle, current approaches rely on fabricating and measuring large amounts of samples with different twist angles, which is not only a tedious process but also limited by the difficulties to identify the exact stacking angle. More importantly, the material quality (e.g., defects, dopants) and interface condition (e.g., surface contaminations) could vary a lot across different samples, posing key technique challenges for accurately investigating the fundamental physics. Although recent report demonstrates in situ twisting of the vdWH using an atomic force microscopy (AFM) tip as the nano-manipulator ${ }^{27}$, it requires complex setup and the $2 \mathrm{D}$ material need to be etched into a designed structure with pushing arm, hindering its practical application as well as fundamental device measurement.

Here, we report a simple approach to fully disassemble the fabricated vdWHs into individual building blocks, by utilizing atomically flat polymers as the handling substrate. The disassembled individual layers can be further reassembled into new $\mathrm{vdWHs}$ with distinct material combinations, sequences, and twisting angles, and hence diverse device functions. With this technique, we demonstrate the same $\mathrm{MoS}_{2}$ flake can be reconfigured from n-type to $\mathrm{p}$-type transistors or from back-gate into top-gate device structures, by re-stacking different contact metals and dielectrics. Furthermore, this method could be extended to multi-layer vdWHs, where a four-layer (graphene/BN/MoS $2 /$ metal) non-volatile memory is successfully disassembled and restacked with a different sequence, and the device function is reconfigured into a Schottky diode. These highly reconfigurable devices are intrinsically different from conventional electronic devices, in which the essential building blocks (e.g., contact, dielectric or barrier layer) are strongly bonded (through covalent or ionic bonds) once deposited and device functions are fixed once fabricated. Finally, we demonstrate a vdWH can be disassembled and re-twisted multiple times of any designed angles with twisting resolution $<0.1^{\circ}$, leading to the observation of highly switchable anisotropic behaviors. Our results not only demonstrate the possibility of re-stacking diverse material building blocks at atomic level with reconfigurable functions, but also offer a general method to manipulate twistable electronics with ultrahigh resolution while ensuring identical materials quality. These proof-of-concept devices could also intrigue a reverse engineering concept of disassembled vdWHs electronics or reassembled vdWHs electronics in parallel of state-of-the-art vdWHs electronics, providing rich implications for multi-functional pluggable electronics, optoelectronics with limited material building blocks.

\section{Results}

Disassembling and reassembling processes of bilayer vdWH. Figure 1 shows the standard disassembling and reassembling processes, using a bilayer vdWH as an example. First, a few-layer $\mathrm{MoS}_{2}$ flake $(\sim 4 \mathrm{~nm}$ thick) is mechanically exfoliated on top of atomically flat polyvinyl alcohol (PVA) substrate (Fig. 1a). Next, another $\mathrm{WSe}_{2}$ flake ( $\sim 5 \mathrm{~nm}$ thick) is dry transferred on top of $\mathrm{MoS}_{2}$ using PVA handling substrate, resulting in a typical $\mathrm{MoS}_{2} /$ $\mathrm{WSe}_{2}$ vdWH, as shown in Fig. 1b. Here, the atomically flat PVA substrate could ensure the intimate contact and strong interaction between substrates and 2D materials ${ }^{28,29}$ (compared to the weak vdW force within $\mathrm{MoS}_{2} / \mathrm{WSe}_{2}$ heterostructure), which is essential to avoid the $\mathrm{MoS}_{2} /$ substrate separation during following disassembling process. This is in great contrast to conventional transfer techniques using polydimethylsiloxane (PDMS) ${ }^{30}$ or polypropylene carbonate (PPC) ${ }^{31}$ as the handling polymers, where the whole vdWH stack would be either left on the substrate or picked up by the polymer, as schematically illustrated in Supplementary Fig. 1. The detailed fabrication process of polymer with atomically flat surface is shown in Methods and Supplementary Fig. 2. Once forming vdWH, the top $\mathrm{WSe}_{2}$ can be separated from the bottom $\mathrm{MoS}_{2}$ by mechanically removing its PVA handling polymer substrate (Fig. 1c), due to the weaker vdW bonding between $\mathrm{MoS}_{2}$ and $\mathrm{WSe}_{2}$ (compared to stronger bonding within $\mathrm{MoS}_{2}$ and PVA substrate). After the disassembling process, the $\mathrm{MoS}_{2}$ still demonstrates flat surface similar to pristine $\mathrm{MoS}_{2}$, as shown AFM measurement in Fig. 1f, g. This observation is resulted from the dry disassembling process used here, without involving any wet chemicals, surface contaminations, or polymer residues, mimicking a reverse engineering of the dry-transfer process. With pristine surface, the disassembled $\mathrm{MoS}_{2}$ can be further used as an individual building block to form new vdWHs. As shown in Fig. 1d, e, another layer of BN could be vdW integrated on the same $\mathrm{MoS}_{2}$ flake to form $\mathrm{BN} / \mathrm{MoS}_{2}$ heterostructure, and then mechanically disassembled again using previous described method. The disassembling and reassembling processes are highly reliable, where the $\mathrm{MoS}_{2}$ still demonstrates fresh surface after ten repetitive cycles, as shown in the AFM image in Fig. $1 \mathrm{~h}$. We note the method is not only limited to bilayer vdWHs, but could be well extended to multi-layer devices or more complicated structures by involving state-of-the-art pickup transfer technique, and will be discussed in detail.

Reconfigurable $\mathrm{MoS}_{2}$ devices with different contact metals. The ability to disassemble the fabricated vdWHs into pristine individual layers, could enable unique reconfigurable transistors by hotplugging different vdW metals as contact electrode and insulating materials as gate dielectric. For example, using a $\mathrm{MoS}_{2}$ flake as the semiconductor channel and $\mathrm{BN} / \mathrm{SiO}_{2}(60 \mathrm{~nm} / 300 \mathrm{~nm}$ thick) as the back-gate dielectric, typical n-type transistor behavior is observed by vdW assembling $\mathrm{Ag}$ metal pairs as the electrodes ${ }^{32}$ (Fig. 2a). As shown in Fig. 2b, the assembled transistor demonstrates n-type $I_{\mathrm{ds}}-V_{\mathrm{gs}}$ transfer curve with on-off ratio over $10^{9}$, as well as linear $I_{\mathrm{ds}}-V_{\mathrm{ds}}$ output characteristics (Fig. 2b, inset), suggesting the ohmic contact between low work function $\mathrm{Ag}$ and underlying $\mathrm{MoS}_{2}$, consistent with previous reports ${ }^{33-35}$. In particular, the threshold voltage of the n-type transistor is close to 0 (Fig. 2b), indicating the optimized interface during vdW integration without additional doping effect. Next, the Ag electrode pairs are mechanically separated from $\mathrm{MoS}_{2}$ channel using the disassembling technique (Fig. 2c), where another pair of pre- 


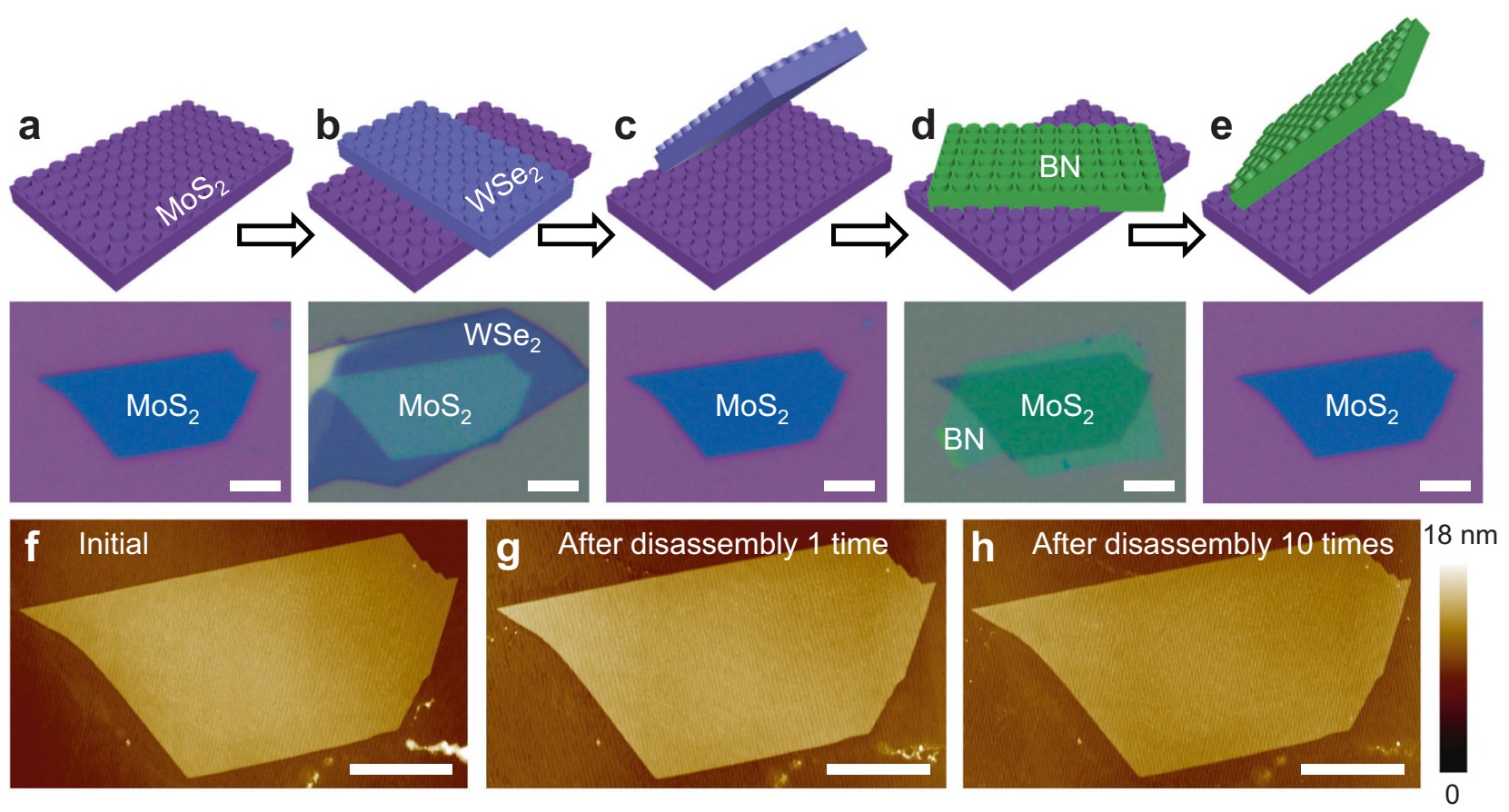

Fig. 1 Characterization of the disassembling/reassembling processes of a bilayer vdWH. a-e Schematics and the optical images of the disassembling/ reassembling processes: $\mathrm{MoS}_{2}$ exfoliation on PVA substrate (a), WSe 2 dry transferred on top of $\mathrm{MoS}_{2}$ (b), WSe $\mathrm{b}_{2}$ physically disassembled from MoS 2 surface (c), BN dry transferred onto the pre-disassembled $\mathrm{MoS}_{2}(\mathbf{d})$, and BN disassembled from $\mathrm{MoS}_{2}$ after 10 times (e). f AFM image of the as-exfoliated $\mathrm{MoS}_{2}$ with pristine surface. $\mathbf{g}$, h AFM images of the $\mathrm{MoS}_{2}$ surface after 1 time (g) and 10 times $(\mathbf{h})$ disassembling/reassembling processes. The clean and pristine $\mathrm{MoS}_{2}$ surface observed here is the result of dry disassembling process, and is essential for further vdW integration. Scale bars, $5 \mu \mathrm{m}$.

fabricated Pt electrodes could be reassembled on the same $\mathrm{MoS}_{2}$ channel using vdW integration technique ${ }^{35}$ (Fig. 2d). The high work function of $\mathrm{Pt}(5.7 \mathrm{eV})$ electrodes matches well with the valance band of $\mathrm{MoS}_{2}$, hence the majority carrier type can be switched from electron to hole ${ }^{35-37}$. As shown in Fig. 2e, using the same $\mathrm{MoS}_{2}$ flake as the channel, the device is reconfigured into well-behaved p-type transistor with linear $I_{\mathrm{ds}}-V_{\mathrm{ds}}$ output curve, suggesting the p-type ohmic contact within vdW $\mathrm{MoS}_{2} / \mathrm{Pt}$ junctions (Fig. 2e, inset). Importantly, the threshold voltage of the reconfigured transistor is also close to 0 , indicating the $\mathrm{vdW}$ disassembling and re-integrating processes could maintain the intrinsic properties of $\mathrm{MoS}_{2}$ channel. We note the vdW integration of different metal electrodes here is not only important to achieve atomically flat metal/MoS ${ }_{2}$ interfaces to avoid the Fermi level pinning effect and to achieve the polarity control, but also essential to achieve weakly bonded metal/ $\mathrm{MoS}_{2}$ contact for successfully disassembling process. This is intrinsically different from conventional transistor with strongly bonded metal/semiconductor interfaces (typically achieved through high energy sputtering or vacuum deposition), where the electrodes can not be removed once deposited and the device function (or transistor polarity) is fixed once fabricated $35,38,39$.

Using this technique, the as-measured p-type transistor is disassembled again (Fig. 2f), and the pristine $\mathrm{MoS}_{2}$ channel could be further integrated with top-gate stacks, resulting in the tunable device geometry from back-gate device to dual-gate structure. As shown in Fig. $2 \mathrm{~g}$, the top-gate stack is pre-fabricated on sacrifice wafer, consisting top-gate dielectric $(20 \mathrm{~nm} \mathrm{BN})$ and gate electrodes $(\mathrm{Ag} / \mathrm{Au}, 30 / 20 \mathrm{~nm})$. After the gate stack is laminated on the previous disassembled $\mathrm{MoS}_{2}$ channel (with pristine surface) (Fig. 2h), the dual-gate transistor electrical measurement is conducted immediately. Figure $2 \mathrm{i}$ shows n-type $I_{\mathrm{ds}}-V_{\mathrm{tg}}$ transfer curve of the device, where the top-gate strongly modulates the channel region. The inset shows 2D plot of the $I_{\mathrm{ds}}$ as a function of top-gate voltage $\left(V_{\mathrm{tg}}\right)$ and back-gate voltage $\left(V_{\mathrm{bg}}\right)$, from which we can determine the slope of threshold voltage shift. The slope gives the ratio between top-gate and back-gate capacitances, $C_{\mathrm{tg}} C_{\mathrm{bg}}$ 17. Using the back-gate capacitance value of $C_{\mathrm{bg}}$ of $9.6 \mathrm{nF} \mathrm{cm}^{-2}$ $\left(300 \mathrm{~nm} \mathrm{SiO}{ }_{2}\right.$ and $\left.60 \mathrm{~nm} \mathrm{BN}\right)$, the top-gate capacitance is calculated to be $163.2 \mathrm{nF} \mathrm{cm}^{-2}$. This corresponds to a $21.7 \mathrm{~nm}$ thick BN, consistent with top-BN used here $(20 \mathrm{~nm}$ identified by AFM and optical contrast), further indicating the well-behaved dual-gate device functions of reassembled vdWHs.

Moreover, we have also performed the AFM measurement of the $\mathrm{MoS}_{2}$ surface after disassembling both Ag electrodes and Pt electrodes on the same $\mathrm{MoS}_{2}$ channel, as shown in Supplementary Fig. 3. The $\mathrm{MoS}_{2}$ channel still demonstrates pristine surface after continuous assembling and disassembling both $\mathrm{Ag}$ and $\mathrm{Pt}$ electrodes, further confirming the mechanical assembling/disassembling approach without contaminations and interfaces residues.

Reliability and stability of the disassembling technique. To confirm the high reliability of our assembling/disassembling processes, we have disassembled/reassembled a $\mathrm{MoS}_{2}$ transistor 10 times by repeatedly vdW integrating/releasing $\mathrm{Ag}$ electrode pairs on the same $\mathrm{MoS}_{2}$ channel. The optical images after every assembling/disassembling process are shown in Supplementary Fig. 4, and the channel region does not show observable damages after ten repetitive processes. Importantly, the electrical measurement is also directly conducted after each assembling process, where the reliability and stability of the assembling/disassembling approach could be evaluated.

The $I_{\mathrm{ds}}-V_{\mathrm{gs}}$ transfer curve and $I_{\mathrm{ds}}-V_{\mathrm{ds}}$ output curve of the $\mathrm{MoS}_{2}$ transistor after each integration process are shown in Supplementary Fig. 5a-j, from which the key device parameters could be extracted. The relationship of two-terminal mobility with different assembling times is demonstrated in Supplementary Fig. 5k, where the extracted values are relatively consistent 

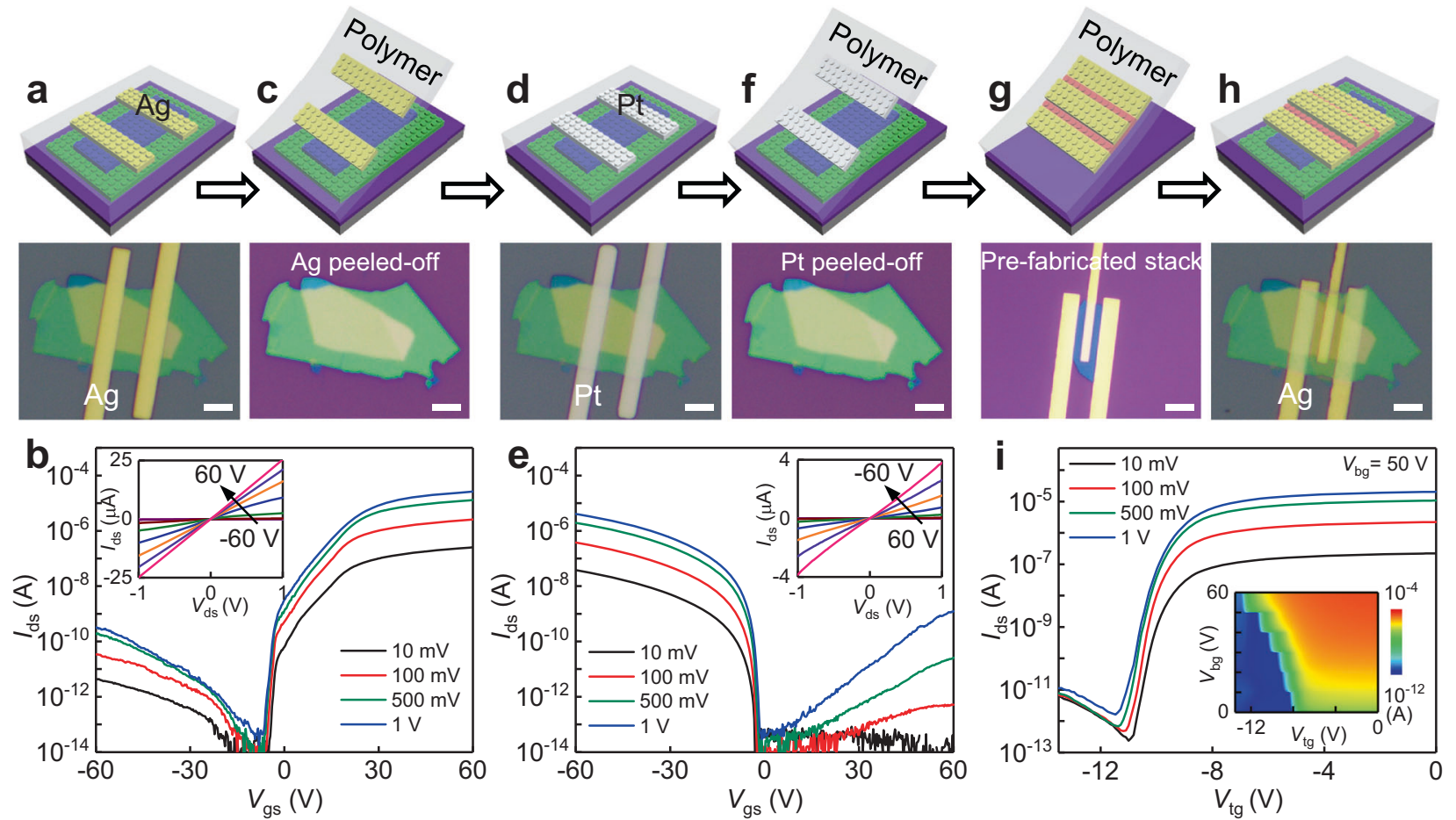

Fig. 2 Reconfigurable transistors by vdW disassembling and integrating various contact metals and dielectric on the same $\mathbf{M o S}_{\mathbf{2}}$ channel. a $\mathrm{Schematic}$ and the optical image of $\mathrm{Ag}$ electrode pairs vdW laminated onto a $\mathrm{MoS}_{2}$ channel. $\mathbf{b} I_{\mathrm{ds}}-V_{\mathrm{gs}}$ transfer characteristics and $I_{\mathrm{ds}}-V_{\mathrm{ds}}$ (inset) output curve of the $\mathrm{MoS}_{2}$ transistor with vdW Ag electrodes, demonstrating n-type device characteristic with ohmic contact. c Schematic and the optical image of Ag electrode pairs physically disassembled from $\mathrm{MoS}_{2}$ surface. The disassembled $\mathrm{MoS}_{2}$ demonstrates clean surface that is essential for further integration. d Schematic and the optical image of Pt electrode pairs vdW laminated on the pre-disassembled $\mathrm{MoS}_{2}$ channel. e $I_{\mathrm{ds}}-V_{\mathrm{gs}}$ transfer characteristics and $I_{\mathrm{ds}}-V_{\mathrm{ds}}$ (inset) output curve of the $\mathrm{MoS}_{2}$ transistor with vdW Pt electrodes, demonstrating the switch of device polarity (from n-type to p-type) simply using different contact metals, due to energy match between high work function Pt and the valance band of $\mathrm{MoS}_{2}$. $\mathbf{f}$ Schematic and the optical image of Pt electrode pairs physically disassembled again from $\mathrm{MoS}_{2}$ surface. $\mathbf{g}$, $\mathbf{h}$ Top-gate stack (consisting BN dielectric and $\mathrm{Ag}$ gate metal) and contact electrode pairs $(\mathrm{Ag})$ are pre-fabricated $(\mathbf{g})$, and vdW laminated onto pre-disassembled $\mathrm{MoS}_{2}$ channel $(\mathbf{h})$, leading to the fabrication of dual-gate device with same $\mathrm{MoS}_{2}$ channel. $\mathbf{i}$ The device demonstrates n-type behavior with dual-gate modulation, and the inset shows two-dimensional plot of the $I_{\mathrm{ds}}$ as a function of $V_{\mathrm{tg}}$ and $V_{\mathrm{bg}}$. Scale bars are $5 \mu \mathrm{m}$ in optical images.

around $50 \mathrm{~cm}^{2} \mathrm{~V}^{-1} \mathrm{~s}^{-1}$ for each measurement (in the range between 45 to $\left.57 \mathrm{~cm}^{2} \mathrm{~V}^{-1} \mathrm{~s}^{-1}\right)$. Similarly, other key device parameters (on-off ratio, on-state resistance, hysteresis) are also summarized in Supplementary Fig. 5l-n, and these figure-ofmerit parameters also remain stable between different assembling processes, indicating relatively high reliability and stability of our assembling/disassembling processes. We note the finite cycle-tocycle variations could originate from the variation of channel location during each integration process, since the alignment transfer techniques have a typical accuracy around $1 \mu \mathrm{m}$ and the exactly same channel location can't be ensured each time; or could be attributed to the variation of Ag electrodes quality (such as its bottom surface roughness or crystallinity).

Reconfigurable electronic devices of a four-layer vdWH. Beyond reassembling and re-plugging into various combinations in bilayer vdWHs, this technique can be applied to multi-layer vdWHs by changing the stacking sequence using same building blocks. For example, we create a four-layer vdWH (graphene/BN/ $\mathrm{MoS}_{2} / \mathrm{Ag}$ ) memory device ${ }^{40}$ on PVA substrate using vdW integration techniques (detailed in Methods and Supplementary Fig. 6), where the graphene, $\mathrm{BN}, \mathrm{MoS}_{2}$, and $\mathrm{Ag}$ are used as floating gate, tunneling dielectric, semiconductor channel, and metal contact, respectively (Fig. 3a). The device shows a large memory window of $90 \mathrm{~V}$ by using back control gate, as shown in Fig. 3b. Furthermore, the program and erase states show a remarkable program/erase $(\mathrm{P} / \mathrm{E})$ current ratio exceeding $10^{5}$, allowing easy readout of the device state. Importantly, the large $\mathrm{P} / \mathrm{E}$ ratio is retained after over $3000 \mathrm{~s}$ measurement, indicating the high durability of the fabricated vdWH memory.

Furthermore, applying the demonstrated disassembling technique, we could separate the four-layer vdWH (with the sequence of graphene/BN/MoS $2 / \mathrm{Ag}$ ) into two individual bilayer vdWHs, where one composes graphene/BN and the other is $\mathrm{MoS}_{2} / \mathrm{Ag}$, as shown in Fig. 3c. The separation of four-layer vdWHs is well-controlled to happen only within middle $\mathrm{BN} / \mathrm{MoS}_{2}$ interface rather than in bottom graphene/BN or top $\mathrm{MoS}_{2} / \mathrm{Ag}$ interfaces, by carefully designing the interaction area (hence the vdW forces) of each vdW interface, as shown in Supplementary Fig. 6. Next, the disassembled building block graphene/BN is flipped upside down into $\mathrm{BN} /$ graphene and stacked with the disassembled $\mathrm{MoS}_{2} / \mathrm{Ag}$ again, therefore the stacking sequence is changed into $\mathrm{BN} /$ graphene/ $\mathrm{MoS}_{2} / \mathrm{Ag}$ with essentially same 2D flakes, as shown in Fig. 3d. This new device structure can function as a Schottky diode, where the BN serves as substrate dielectric, graphene and $\mathrm{MoS}_{2}$ form PN diode and $\mathrm{Ag}$ is used as the electrical contact of $\mathrm{MoS}_{2}$. Due to the Fermi level difference between graphene and n-type $\mathrm{MoS}_{2}$, the device shows well-behaved n-type Schottky diode function with rectification ratio over $10^{2}$ (Fig. 3e), consistent with previous reports ${ }^{41,42}$. We note the demonstrated reconfigurable devices from memory to diode only represent two different sequences (out of 12 different sequences using a four-layer vdWH), and more device functions are expected if re-sequenced into different device 

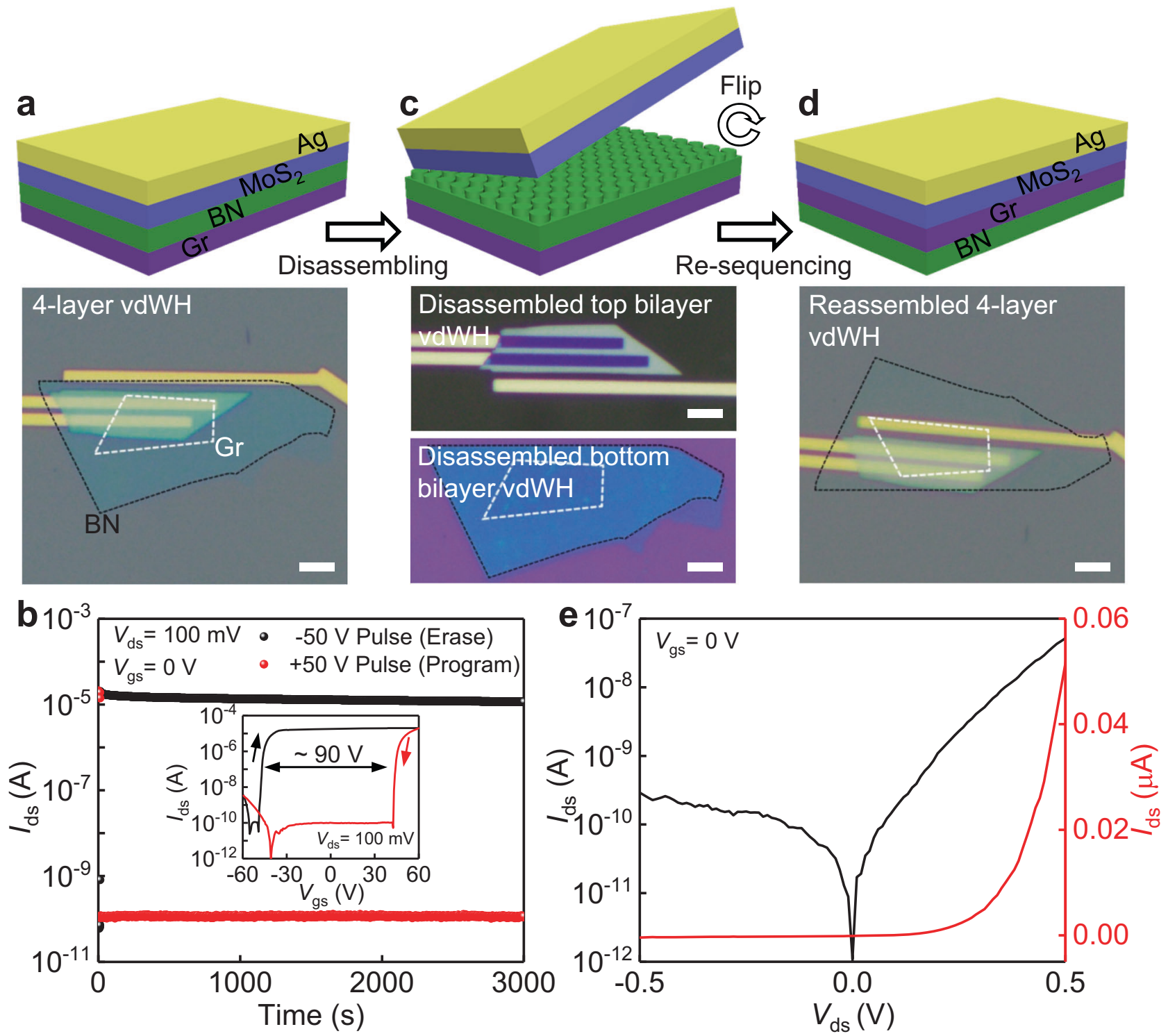

Fig. 3 Reconfigurable electronic devices by disassembling and reassembling a four-layer vdWH. a Schematic and the optical image of a floating gate transistor using a four-layer vdWH of graphene/BN/MoS $/ \mathrm{Ag}$, where the graphene $(\mathrm{Gr}), \mathrm{BN}, \mathrm{MoS}_{2}$, and $\mathrm{Ag}$ are used as floating gate, tunneling dielectric, semiconductor channel, and metal contact, respectively. b Retention performance of the floating gate transistor (initial four-layer vdWH) and the inset shows transfer characteristics of the floating gate transistor. c Schematic and the optical images of four-layer vdWH separated into two individual bilayer $\mathrm{vdWHs}\left(\mathrm{MoS}_{2} / \mathrm{Ag}\right.$ in top and graphene/BN in bottom). d Schematic and the optical image of a reassembled four-layer vdWH with a new sequence (BN/graphene/ $\mathrm{MoS}_{2} / \mathrm{Ag}$ ) using the same building blocks, representing a Schottky diode device function. e Output characteristics (semi-log and linear plots) of the reassembled four-layer vdWH diode. Scale bars are $5 \mu \mathrm{m}$ in optical images.

structures, such as $\mathrm{Ag} / \mathrm{graphene} / \mathrm{BN} / \mathrm{MoS}_{2}$ tunneling transistor ${ }^{43}$, or $\mathrm{Ag} / \mathrm{BN} / \mathrm{MoS}_{2} /$ graphene photodetectors ${ }^{44,45}$. The choice of stacking sequences (hence device structures and functions) could increase exponentially with more layer numbers, offering another degree of freedom for constructing multi-functional vdWHs devices using same material building blocks.

Reconfigurable anisotropic Raman behaviors. Finally, besides re-combination and re-sequencing, the disassembled building blocks could be stacked again with different angles, leading to controllable twistable electronics with high twisting resolution. To demonstate this, two layered materials germanium arsenide (GeAs, $\sim 25 \mathrm{~nm}$ thick) and black phosphorus (BP, $\sim 10 \mathrm{~nm}$ thick) are used, and both materials demonstrate highly anisotropic lattice structures. The GeAs/BP vdWH is stacked using stardard dry-alignment transfer setup (see Methods). After disassembling the vdWH, the bottom BP and its holding substrate are rotated with any desired angle by a motor-controlled rotator with a resolution $\sim 0.1^{\circ}$ (Fig. 4a-e). As demonstrated in Fig. $4 \mathrm{f}-\mathrm{k}$ and Supplementary Fig. 7, we have re-twisted the vdWH 16 times with a rotation spacing of $22.5^{\circ}$. We could measure the Raman spectrum of the vdWH under every twist angle, leading to the observation of switchable anisotropic behaviors within vdWHs. For example, when the GeAs and BP are alinged at 0 degree (alignment between the zigzag direction ${ }^{46,47}$ ), the $\mathrm{vdWH}$ demonstates a typical fourfold anisotropic Raman behavior, as shown in Fig. 4l. When the two materials are rotated to $22.5^{\circ}$ and $45^{\circ}$, the symmetry of Raman signal is broken owing to the misalignment of two lattice structures, where the vdWHs exhibit eightfold anisotropic Raman signals, as shown in Fig. $4 \mathrm{~m}$ and 

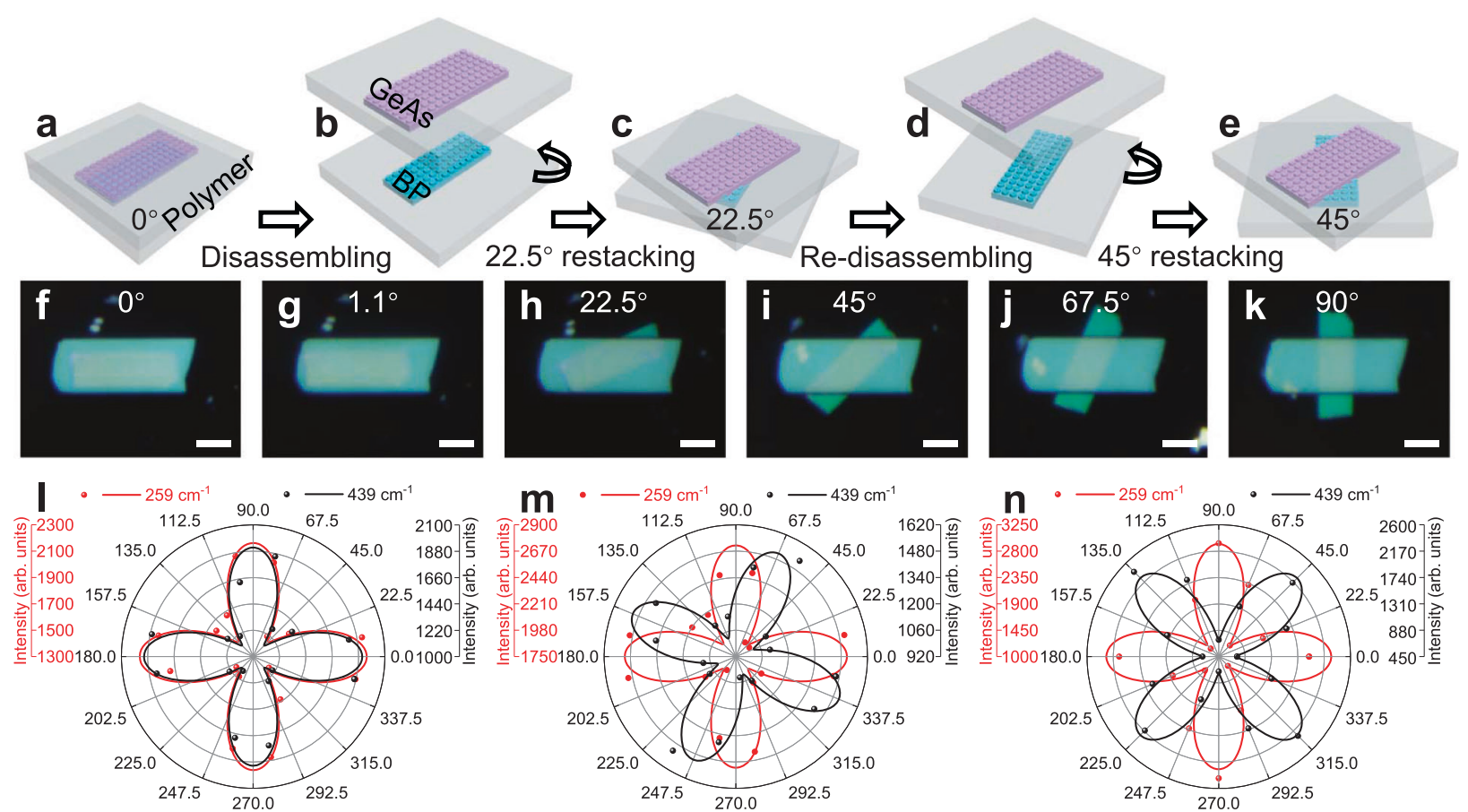

Fig. 4 Disassembling and re-twisting of vdWHs. a-e Schematic illustration of the fabrication process of GeAs/BP vdWHs with different twist angles with five steps: the stacked GeAs/BP heterostructure (a); GeAs separated from BP (b); BP rotated $22.5^{\circ}$ and re-stacked to form GeAs/BP heterostructure (c); GeAs separated from BP again (d); BP rotated $45^{\circ}$ and re-stacked into GeAs/BP heterostructure (e). f-k Optical images of GeAs/BP vdWHs with various twist angles of $0^{\circ}(\mathbf{f}), 1.1^{\circ}(\mathbf{g}), 22.5^{\circ}(\mathbf{h}), 45^{\circ}(\mathbf{i}), 67.5^{\circ}(\mathbf{j})$, and $90^{\circ}(\mathbf{k})$. Scale bars are $5 \mu \mathrm{m}$. I-n Polar plots of Raman intensity of GeAs/BP heterostructures with various twist angles of $0^{\circ}(\mathbf{I}), 22.5^{\circ}(\mathbf{m})$ and $45^{\circ}(\mathbf{n})$

Fig. 4n, respectively. The polarized Raman spectra of GeAs/BP heterostructures with various twist angles are shown in Supplementary Fig. 8.

In particular, we have also demonstrated a rotation angle of $1.1^{\circ}$ (Fig. 4g), which is reocongnized as magic angle in twisted bilayer graphene ${ }^{21,22,48}$. Although we do not observe novel physics in this stacking angle (between GeAs and BP), the highly controllable rotation technique could offer a new approch for investigating the rich physics within twistable electronics. Previous twistable electronics are largely achieved through two different approaches: one relies on fabricating and measuring large amounts of samples with different twist angles ${ }^{24,49}$, and the other relies on in situ mechanically pushing the etched 2D materials using AFM tip ${ }^{27}$. Although these approaches have successfully demonstrated interesting physics beyond the reach of existing materials, they are still limited by the complex fabrication and twisting process, as well as the highly variable material qualities (e.g., defects, dopants) and interface conditions (e.g., air bubble, surface contaminations) across different samples ${ }^{50-52}$. Within our simple approach, identical material quality is always ensured (because of the same material building blocks used) and the twisting angle is well-controlled. Based on this, the impact of small rotation angle change (e.g., change from $1.1^{\circ}$ to $1.15^{\circ}$ ) to the lattice commensuration or moiré pattern generation would be an interesting topic for further investigation, which is difficult to achieve using previous techniques. We note the rotation resolution (currently $0.1^{\circ}$ ) could also be improved with better motor rotator, which could reach an ultra-high resolution below $0.01^{\circ}$ in commercialized products.

\section{Discussion}

In summary, we report a general technique for disassembling the artificial stacked vdWHs into individual building blocks, which can be further reassembled into new vdWHs via re-combining, re-sequencing, and re-twisting, hence new device functions. This method shows high reliability and stability, as verified by the multiple disassembling and reassembling processes and the corresponding AFM measurement. Within this, we have demonstrated reconfigurable transistors functions (n-type to p-type, back-gate to dual-gate) in a bilayer vdWH through re-stacking; reconfigurable device behaviors (floating gate memory to Schottky diode) in a four-layer vdWH through re-sequencing; and reconfigurable anisotropic Raman behaviors (fourfold to eightfold) through re-twisting, using same material building blocks. These proof-of-concept devices not only demonstrate the possibility to manipulate and re-stack diverse material layers at atomic level, but could also intrigue a research field of disassembled/reassembled vdWHs electronics in parallel of state-ofthe-art vdWHs electronics, providing rich implications for multifunctional pluggable electronics, optoelectronics with limited material building blocks.

\section{Methods}

Disassembling process of a bilayer vdWH. First, thin PVA layer ( $\sim 8 \mathrm{~nm}$ thick) is spin-coated on $\mathrm{SiO}_{2}$ substrate, where a $\mathrm{MoS}_{2}$ flake can be further mechanically exfoliated on top. The spin-coated PVA is atomically flat, with a root-mean-square surface roughness of $0.25 \mathrm{~nm}$ (Supplementary Fig. 9). It is noted that the PVA layer is essential here to fix the peeled $\mathrm{MoS}_{2}$, owing to its high adhesion forces. Next, a few-layer $\mathrm{WSe}_{2}$ or $\mathrm{BN}$ ( $\sim 6 \mathrm{~nm}$ or $32 \mathrm{~nm}$, respectively) is mechanically exfoliated on top of sacrifice $\mathrm{SiO}_{2}$ substrate and then functionalized by a hexamethyldisilazane (HMDS) layer in a sealed chamber at $80^{\circ} \mathrm{C}$ for about $5 \mathrm{~min}$. Next, the wafer is covered by a PVA layer ( $2 \mu \mathrm{m}$ thick) through spin-coating, where the PVA layer (used as the handling substrate) with $\mathrm{WSe}_{2}$ or $\mathrm{BN}$ wrapped underneath is mechanically released and transferred on top of $\mathrm{MoS}_{2}$. Once the vdWH formed, the PVA layer can be further removed (together with $\mathrm{WSe}_{2}$ or $\mathrm{BN}$ wrapped underneath) using thermal release tape, representing the disassembling process of the vdWH.

Fabrication process of a reconfigurable $\mathrm{MoS}_{2}$ device with different contact metals. First, a few-layer $\mathrm{MoS}_{2}$ is exfoliated on $300 \mathrm{~nm} \mathrm{SiO} 2 / \mathrm{p}^{++} \mathrm{Si}$ substrate (covered with a thin layer of BN). Next, metal electrodes (50 nm thick Pt or 30/20 
$\mathrm{nm}$ thick $\mathrm{Ag} / \mathrm{Au}$ ) are pre-fabricated on sacrificial substrate through electron-beam evaporation, which can be mechanically released from the substrate and physically laminated on $\mathrm{MoS}_{2}$, resulting in an atomically sharp and clean metal-2D material interface. After that, the poly(methyl methacrylate) (PMMA) on top of the metal pads is removed using electron-beam lithography and development processes for electrical probing and measurements.

To disassembled the vdWH, the transferred metal electrodes are mechanically separated from the $\mathrm{MoS}_{2}$ channel using a thermal release tape. We note that the PMMA on top of BN is removed using standard electron-beam lithography and development processes before the disassembling process to prevent the channel material being taken away. Pre-fabricated Pt electrodes and top-gate stacks are laminated and disassembled using the same method described above.

\section{Disassembling and reassembling processes of a four-layer vdWH. First,} monolayer graphene is mechanically exfoliated onto PVA $(\sim 8 \mathrm{~nm}) / \mathrm{SiO}_{2}(300 \mathrm{~nm})$ substrate, and then $13 \mathrm{~nm}$ thick $\mathrm{BN}$ is dry transferred onto as-exfoliated graphene, leading to the formation of graphene/BN vdWH. Next, $\mathrm{MoS}_{2} / \mathrm{Ag}$ vdWH is prefabricated on top of a sacrificial substrate, which can be further mechanically released and laminated onto the graphene/BN vdWH, resulting in a four-layer (graphene/BN/MoS $2 / \mathrm{Ag}$ ) vdW non-volatile memory.

To disassemble the four-layer vdWH, the upper two layers $\left(\mathrm{MoS}_{2} / \mathrm{Ag}\right)$ are mechanically separated from $\mathrm{BN}$ by using thermal release tape to pick up PMMA layer. The PMMA layer (together with the $\mathrm{MoS}_{2} / \mathrm{Ag}$ vdWH) is further transferred onto a $\mathrm{SiO}_{2}$ substrate by removing thermal release tape under $100^{\circ} \mathrm{C}$. Next, $500 \mathrm{~nm}$ thick PMMA is spin-coated on the bottom vdWH (graphene/BN), and the substrate is immersed into an $80^{\circ} \mathrm{C}$ deionized water bath for $30 \mathrm{~min}$ to dissolve the PVA underneath. Once PVA is dissolved, the PMMA membrane will float on top of water, which can be further flipped and fished using a Si wafer (with $100 \mathrm{~nm}$ thick $\mathrm{SiO}_{2}$ ). After that, the substrate is heated to $120^{\circ} \mathrm{C}$ for $10 \mathrm{~min}$ to fully remove any water adsorbed on BN/graphene vdHW. Finally, the pre-disassembled $\mathrm{MoS}_{2} /$ $\mathrm{Ag}$ is re-stacked onto the $\mathrm{BN} /$ graphene, resulting in a four-layer (BN/graphene/ $\left.\mathrm{MoS}_{2} / \mathrm{Ag}\right) \mathrm{vdW}$ diode. The corresponding schematics and optical images are shown in Supplementary Fig. 6.

Disassembling and re-twisting of vdWH. Multi-layer GeAs and BP are mechanically exfoliated on two sacrifice wafers, respectively. Next, the wafers are functionalized by HMDS and spin-coated with a PMMA layer, where the PMMA layer with BP wrapped underneath is picked up using transparent double-sided tape, and the tape with the BP side up is fixed on a slide glass. Afterward, the PMMA layer with GeAs wrapped underneath is mechanically released and transferred onto BP. Subsequently, the PMMA layer with GeAs can be mechanically separated from BP using Scotch tape, placed on PDMS and transferred onto $\mathrm{BP}$ again under optical microscope. GeAs/BP vdWHs with various twist angles can be achieved by repeating the above approach multiple times, as shown in Sup plementary Fig. 7. Besides, PVA could also be used as the handling substrate for retwisting electronics. As shown in Supplementary Fig. 10, we have used PVA substrate to fabricate twisted GeAs/BP vdWHs with same fabrication process, except PMMA is changed into PVA.

Materials characterizations and electrical measurements. An atomic force microscope (Bioscope system, Brucker) is used to characterize the morphology and thickness. Raman spectrum studies of GeAs/BP vdWHs are conducted using a confocal microscope (Renishaw invia-reflex) excited by $633 \mathrm{~nm}$ laser and angle dependent polarized Raman spectra are obtained by precisely rotating sample orientation. Electrical measurements are carried out in probe station (Lakeshore, PS-100) at room temperature, using Keysight B2912A source measurement unit and Agilent B1500A semiconductor analyzer.

\section{Data availability}

The data that support the findings of this study are available from the corresponding author upon reasonable request.

Received: 10 November 2020; Accepted: 3 March 2021;

Published online: 23 March 2021

\section{References}

1. Dean, C. R. et al. Boron nitride substrates for high-quality graphene electronics. Nat. Nanotechnol. 5, 722-726 (2010).

2. Britnell, L. et al. Field-effect tunneling transistor based on vertical graphene heterostructures. Science 335, 947-950 (2012).

3. Geim, A. K. \& Grigorieva, I. V. Van der Waals heterostructures. Nature 499, 419-425 (2013).

4. Lee, C. H. et al. Atomically thin p-n junctions with van der Waals heterointerfaces. Nat. Nanotechnol. 9, 676-681 (2014).
5. Withers, F. et al. Light-emitting diodes by band-structure engineering in van der Waals heterostructures. Nat. Mater. 14, 301-306 (2015).

6. Novoselov, K. S., Mishchenko, A., Carvalho, A. \& Castro Neto, A. H. 2D materials and van der Waals heterostructures. Science 353, aac9439 (2016).

7. Liu, Y. et al. Van der Waals heterostructures and devices. Nat. Rev. Mater. 1, 16042 (2016).

8. Jariwala, D., Marks, T. J. \& Hersam, M. C. Mixed-dimensional van der Waals heterostructures. Nat. Mater. 16, 170-181 (2017).

9. Wang, Q. et al. Nonvolatile infrared memory in $\mathrm{MoS}_{2} / \mathrm{PbS}$ van der Waals heterostructures. Sci. Adv. 4, eaap7916 (2018).

10. Liang, S. J., Cheng, B., Cui, X. \& Miao, F. Van der Waals heterostructures for high-performance device applications: challenges and opportunities. $A d v$. Mater. 32, 1903800 (2019).

11. Liu, Y., Huang, Y. \& Duan, X. Van der Waals integration before and beyond two-dimensional materials. Nature 567, 323-333 (2019).

12. Yankowitz, M., Ma, Q., Jarillo-Herrero, P. \& LeRoy, B. J. van der Waals heterostructures combining graphene and hexagonal boron nitride. Nat. Rev. Phys. 1, 112-125 (2019).

13. Rivera, P. et al. Observation of long-lived interlayer excitons in monolayer $\mathrm{MoSe}_{2}-\mathrm{WSe}_{2}$ heterostructures. Nat. Commun. 6, 6242 (2015).

14. Unuchek, D. et al. Room-temperature electrical control of exciton flux in a van der Waals heterostructure. Nature 560, 340-344 (2018).

15. Seyler, K. L. et al. Valley manipulation by optically tuning the magnetic proximity effect in $\mathrm{WSe}_{2} / \mathrm{CrI}_{3}$ heterostructures. Nano Lett. 18, 3823-3828 (2018).

16. Chen, W. et al. Direct observation of van der Waals stacking-dependent interlayer magnetism. Science 366, 983-987 (2019).

17. Yankowitz, M. et al. Tuning superconductivity in twisted bilayer graphene. Science 363, 1059-1064 (2019).

18. Roy, K. et al. Graphene- $\mathrm{MoS}_{2}$ hybrid structures for multifunctional photoresponsive memory devices. Nat. Nanotechnol. 8, 826-830 (2013).

19. Wang, X. et al. Recent advances in the functional $2 \mathrm{D}$ photonic and optoelectronic devices. Adv. Opt. Mater. 7, 1801274 (2018).

20. Li, B. et al. Probing van der Waals interactions at two-dimensional heterointerfaces. Nat. Nanotechnol. 14, 567-572 (2019).

21. Cao, Y. et al. Correlated insulator behaviour at half-filling in magic-angle graphene superlattices. Nature 556, 80-84 (2018).

22. Cao, Y. et al. Unconventional superconductivity in magic-angle graphene superlattices. Nature 556, 43-50 (2018).

23. Seyler, K. L. et al. Signatures of moire-trapped valley excitons in $\mathrm{MoSe}_{2} / \mathrm{WSe}_{2}$ heterobilayers. Nature 567, 66-70 (2019).

24. Alexeev, E. M. et al. Resonantly hybridized excitons in moire superlattices in van der Waals heterostructures. Nature 567, 81-86 (2019).

25. Jin, C. et al. Observation of moire excitons in $\mathrm{WSe}_{2} / \mathrm{WS}_{2}$ heterostructure superlattices. Nature 567, 76-80 (2019).

26. Tran, K. et al. Evidence for moire excitons in van der Waals heterostructures. Nature 567, 71-75 (2019)

27. Ribeiro-Palau, R. et al. Twistable electronics with dynamically rotatable heterostructures. Science 361, 690-693 (2018).

28. $\mathrm{Li}, \mathrm{Z}$. et al. Efficient strain modulation of $2 \mathrm{D}$ materials via polymer encapsulation. Nat. Commun. 11, 1151 (2020).

29. Huang, Z. et al. Versatile construction of van der Waals heterostructures using a dual-function polymeric film. Nat. Commun. 11, 3029 (2020).

30. Castellanos-Gomez, A. et al. Deterministic transfer of two-dimensional materials by all-dry viscoelastic stamping. 2D Mater. 1, 011002 (2014).

31. Wang, L. et al. One-dimensional electrical contact to a two-dimensional material. Science 342, 614-617 (2013).

32. Yuan, H. et al. Influence of metal- $\mathrm{MoS}_{2}$ interface on $\mathrm{MoS}_{2}$ transistor performance: comparison of $\mathrm{Ag}$ and Ti contacts. ACS Appl. Mater. Interfaces 7, 1180-1187 (2015).

33. Radisavljevic, B., Radenovic, A., Brivio, J., Giacometti, V. \& Kis, A. Single layer $\mathrm{MoS}_{2}$ transistors. Nat. Nanotechnol. 6, 147-150 (2011).

34. Das, S., Chen, H. Y., Penumatcha, A. V. \& Appenzeller, J. High performance multilayer $\mathrm{MoS}_{2}$ transistors with scandium contacts. Nano Lett. 13, 100-105 (2013).

35. Liu, Y. et al. Approaching the Schottky-Mott limit in van der Waals metal-semiconductor junctions. Nature 557, 696-700 (2018).

36. Yu, L. et al. High-performance WSe $e_{2}$ complementary metal oxide semiconductor technology and integrated circuits. Nano Lett. 15, 4928-4934 (2015).

37. Lim, J. Y. et al. Homogeneous $2 \mathrm{D} \mathrm{MoTe} 2 \mathrm{p}-\mathrm{n}$ junctions and CMOS inverters formed by atomic-layer-deposition-induced doping. Adv. Mater. 29, 1701798 (2017).

38. Chen, L. J. Solid state amorphization in metal/Si systems. Mater. Sci. Eng. Rep. 29, 115-152 (2000)

39. Jung, Y. et al. Transferred via contacts as a platform for ideal two-dimensional transistors. Nat. Electron. 2, 187-194 (2019).

40. Choi, M. S. et al. Controlled charge trapping by molybdenum disulphide and graphene in ultrathin heterostructured memory devices. Nat. Commun. 4 , 1624 (2013) 
41. Yu, W. J. et al. Vertically stacked multi-heterostructures of layered materials for logic transistors and complementary inverters. Nat. Mater. 12, 246-252 (2013).

42. Henck, H. et al. Electrolytic phototransistor based on graphene- $\mathrm{MoS}_{2}$ van der Waals p-n heterojunction with tunable photoresponse. Appl. Phys. Lett. 109, 113103 (2016)

43. Li, C. et al. Low sub-threshold swing realization with contacts of graphene/h-BN/ $\mathrm{MoS}_{2}$ heterostructures in $\mathrm{MoS}_{2}$ transistors. Appl. Phys. Lett. 111, 193502 (2017).

44. Huo, N. \& Konstantatos, G. Recent progress and future prospects of 2D-based photodetectors. Adv. Mater. 30, 1801164 (2018).

45. Koppens, F. H. et al. Photodetectors based on graphene, other twodimensional materials and hybrid systems. Nat. Nanotechnol. 9, 780-793 (2014).

46. Yang, S. et al. Highly in-plane optical and electrical anisotropy of 2D germanium arsenide. Adv. Funct. Mater. 28, 1707379 (2018).

47. Kim, J. et al. Anomalous polarization dependence of Raman scattering and crystallographic orientation of black phosphorus. Nanoscale 7, 18708-18715 (2015).

48. Bistritzer, R. \& MacDonald, A. H. Moire bands in twisted double-layer graphene. Proc. Natl Acad. Sci. USA 108, 12233-12237 (2011).

49. Kim, K. et al. Tunable moire bands and strong correlations in small-twistangle bilayer graphene. Proc. Natl Acad. Sci. USA 114, 3364-3369 (2017)

50. Kretinin, A. V. et al. Electronic properties of graphene encapsulated with different two-dimensional atomic crystals. Nano Lett. 14, 3270-3276 (2014).

51. Haigh, S. J. et al. Cross-sectional imaging of individual layers and buried interfaces of graphene-based heterostructures and superlattices. Nat. Mater. 11, 764-767 (2012).

52. $\mathrm{Vu}, \mathrm{Q}$. A. et al. Near-zero hysteresis and near-ideal subthreshold swing in $\mathrm{h}$ $\mathrm{BN}$ encapsulated single-layer $\mathrm{MoS}_{2}$ field-effect transistors. $2 \mathrm{D}$ Mater. 5 , 031001 (2018).

\section{Acknowledgements}

This work was supported by the National Natural Science Foundation of China (Grant Nos. 51991340, 51991341, 51802090, and 61874041).

\section{Author contributions}

Y.L. conceived the research. Y.L. and Q.T. designed the experiments. Q.T. performed the experiments and data analysis. R.W. and X.D. contributed to Raman characterizations.
Q.L., W.L., Liting L., and Lei L. contributed to the device fabrication. Y.C., L.K., and Zheyi L. contributed to discussions and data analysis. J.J. and B.L. contributed to AFM measurement. Zhiwei L. contributed to the PVA substrate fabrication. Y.L. and Q.T. cowrote the paper. All authors discussed the results and commented on the manuscript.

\section{Competing interests}

The authors declare no competing interests.

\section{Additional information}

Supplementary information The online version contains supplementary material available at https://doi.org/10.1038/s41467-021-22118-y.

Correspondence and requests for materials should be addressed to Y.L.

Peer review information Nature Communications thanks Won Jong Yoo and the other anonymous reviewer(s) for their contribution to the peer review of this work.

Reprints and permission information is available at http://www.nature.com/reprints

Publisher's note Springer Nature remains neutral with regard to jurisdictional claims in published maps and institutional affiliations.

\section{(c) (i)}

Open Access This article is licensed under a Creative Commons Attribution 4.0 International License, which permits use, sharing, adaptation, distribution and reproduction in any medium or format, as long as you give appropriate credit to the original author(s) and the source, provide a link to the Creative Commons license, and indicate if changes were made. The images or other third party material in this article are included in the article's Creative Commons license, unless indicated otherwise in a credit line to the material. If material is not included in the article's Creative Commons license and your intended use is not permitted by statutory regulation or exceeds the permitted use, you will need to obtain permission directly from the copyright holder. To view a copy of this license, visit http://creativecommons.org/ licenses/by/4.0/.

(C) The Author(s) 2021 